\title{
Transverse force in a relativistic beam moving along a curved trajectory
}

\author{
Gennady Stupakov® \\ SLAC National Accelerator Laboratory, Menlo Park, California 94025, USA
}

(Received 13 November 2021; accepted 10 January 2022; published 18 January 2022)

\begin{abstract}
When a relativistic beam moves along a curved trajectory it loses energy on coherent radiation. The effect of this radiation on the beam dynamics is described by the so call coherent synchrotron radiation (CSR) longitudinal wake field. Among different models of the CSR wake, the simplest is the onedimensional (1D) one where the beam is treated as a line-charge. The self-field of the beam on a curved trajectory also creates a transverse component of the force. Unfortunately, there is a confusion in the literature as to whether a 1D model can be worked out for the transverse force inside the beam. In this paper, we show how such a 1D model can be consistently derived for a general curvilinear beam orbit if one formulates the equations of motion for the beam particles in Hamiltonian form and uses a renormalized transverse force. Unfortunately, this scheme cannot be applied to the classical problem of relativistic beam passing through a single bending magnet, because the scalar potential in the initial conditions for the Hamiltonian variables cannot be defined in the 1D model. In this case, one should use the 3D transverse force, for which we derive analytical expressions. For the steady state, our calculations show an excellent agreement with computer simulations. We also calculate the transient effects for this force at the entrance to and the exit from the bend. Our results provide a new way to accommodate the transverse force into the existing and new simulation codes which is important for many applications of the high-current, small-emittance relativistic beams.
\end{abstract}

DOI: 10.1103/PhysRevAccelBeams.25.014401

\section{INTRODUCTION}

Self-fields often play an important role in the dynamics of high-current beams of charged particles. When a beam travels along a straight line, such forces are usually associated with the space charge effects [1]. The longitudinal space charge force scales with the beam energy as $\gamma^{-2}$, where $\gamma$ is the Lorentz factor. A longitudinal force also arises when the beam changes its transverse size (e.g., converging toward a final focus in a collider), to keep the balance between the energy of the Coulomb field of the bunch and the kinetic energy of the particles $[2,3]$.

When the beam trajectory is bent by magnetic field, the beam emits electromagnetic radiation which leads to the emerging of the coherent synchrotron radiation (CSR) wake field. The longitudinal radiation reaction force associated with this wake is responsible for the energy balance in the process of the coherent radiation of the bunch. In addition to the longitudinal force, particles of a beam traveling along a curvilinear orbit also experience a transverse force. This force is not caused by the radiation-it exists even for a coasting beam which does not radiate

Published by the American Physical Society under the terms of the Creative Commons Attribution 4.0 International license. Further distribution of this work must maintain attribution to the author(s) and the published article's title, journal citation, and DOI. coherently. The attention to this force was attracted by R. Talman in Ref. [4] whose work initiated several subsequent studies [5-7].

The first studies of the steady-state CSR longitudinal wake were carried out in 1D approximation, that is for a line-charge beam with a vanishing transverse size [8-10]. This 1D model was later extended for the case of a bending magnet of finite length [11,12], and for a beam line with several bending magnets [13-15]. Experimental data from bunch compression at the Linac Coherent Light Source [16] showed that the 1D CSR wakefield model in many cases agrees very well with observations.

It seems natural to try to extend the line-charge beam model and to add to it the transverse force acting on the beam when it moves in a curved trajectory. The first attempt in this direction was made in Ref. [9] where a formula for the steady state transverse force was derived for a line-charge beam moving in a circle. Unfortunately, attempts to expand this analysis to the case of a finite bend [17] were not successfulthe transverse force was calculated only at the locations in front of the beam, while the most interesting would be to find the force acting on the beam particles. Moreover, these attempts initiated a discussion in the literature about the so-called "cancellation" effect between some terms in the expression for the transverse force and "misconceptions" in the treatment of the transverse force $[7,18,19]$.

We give our analysis of the limitations of the 1D model of the transverse force in Sec. II of this paper. We show that 
using a kind of renormalization procedure one indeed can obtain the transverse force for a line charge-beam that in the case of a circular orbit reproduces the result of Ref. [9]. However, if one wants to formulate the initial conditions for particle equations of motion, the transverse size of the beam cannot be neglected. In practical applications one might prefer to work with a $3 \mathrm{D}$ expression for the transverse force that we derive in Sec. III following the approach developed in our earlier paper [20]. In Sec. IV, we simplify the general expression for the force for the case of a circular orbit, and in Sec. V we compare our analytical results with numerically computed transverse force. We also show how to calculate the transient transverse force at the entrance to and the exit from a bending magnet. We conclude this paper with the summary Sec. VI. Some technical details of the derivation are placed in the Appendixes A, B and C.

Throughout this paper we neglect the effect of the metal boundaries near the beam and assume that the beam propagates in free space. We consider a relativistic beam and in most cases use the approximation $v=c$ when we calculate the field. The CGS system of units is used throughout the paper. To convert our formulas for the force to SI units, one has to replace the charge $Q$ (or charge per unit length or per unit volume) by $Q / 4 \pi \epsilon_{0}$ where $\epsilon_{0}$ is the permittivity of free space.

\section{LIMITATIONS OF THE 1D MODEL FOR THE TRANSVERSE FORCE}

Can a 1D model for the transverse force be worked out, similar to the 1D model of the longitudinal CSR wake field? In the 1D model the beam is represented by the charge density per unit length, $\lambda(s-c t)$, where $s$ is the path length measured along the orbit, and we assume that the beam particles move with the speed of light. ${ }^{1}$ The scalar potential $\phi(\boldsymbol{r}, t)$ and the vector potential $\boldsymbol{A}(\boldsymbol{r}, t)$ of such a beam are given by the following formulas:

$$
\begin{aligned}
& \phi(\boldsymbol{r}, t)=\int_{-\infty}^{\infty} \frac{d s^{\prime}}{\left|\boldsymbol{r}-\boldsymbol{r}_{0}\left(s^{\prime}\right)\right|} \lambda\left(s^{\prime}-c t_{\mathrm{ret}}\right), \\
& \boldsymbol{A}(\boldsymbol{r}, t)=\int_{-\infty}^{\infty} \frac{d s^{\prime}}{\left|\boldsymbol{r}-\boldsymbol{r}_{0}\left(s^{\prime}\right)\right|} \boldsymbol{\tau}\left(s^{\prime}\right) \lambda\left(s^{\prime}-c t_{\mathrm{ret}}\right),
\end{aligned}
$$

where $\boldsymbol{r}_{0}(s)$ defines the beam orbit, $t_{\text {ret }}\left(\boldsymbol{r}, s^{\prime}, t\right)=$ $t-\left|\boldsymbol{r}-\boldsymbol{r}_{0}\left(s^{\prime}\right)\right| / c$ is the retarded time, and $\boldsymbol{\tau}$ is the unit tangential vector, $\boldsymbol{\tau}(s)=d \boldsymbol{r}_{0}(s) / d s$. The vector $\boldsymbol{\tau}$ in Eq. (1b) replaced the normalized velocity $\boldsymbol{\beta}=\boldsymbol{v} / c$ which in the $1 \mathrm{D}$ model is directed along the tangent line to the

\footnotetext{
${ }^{1}$ Dropping the assumption $v=c$ would lead to an additional singularity of the fields due to the longitudinal space charge force $\propto \gamma^{-2}$. With some extra effort this singularity can be eliminated through a renormalization procedure introduced in Ref. [11], however, an easier approach is to assume $\gamma=\infty$.
}

orbit. The electric and magnetic fields are expressed through the potentials,

$$
\boldsymbol{E}=-\nabla \phi-\frac{1}{c} \frac{\partial \boldsymbol{A}}{\partial t}, \quad \boldsymbol{H}=\nabla \times \boldsymbol{A} .
$$

Note that if we take the observation point on the beam orbit, $\boldsymbol{r}=\boldsymbol{r}_{0}(s)$, the integrals (1) logarithmically diverge as $s^{\prime} \rightarrow s$ (assuming $\lambda(s-c t) \neq 0$ ), so the potentials are not defined inside the beam. For this reason, in general, the electric and magnetic fields (2) are also singular on the orbit $\boldsymbol{r}_{0}(s)$ inside the beam. However, there is an important combination of $\phi$ and $\boldsymbol{A}$, the longitudinal potential $\boldsymbol{V}$, that can be defined following Ref. [9] everywhere on the orbit,

$$
\begin{aligned}
V(s, t) & =\phi-\boldsymbol{\tau} \cdot \boldsymbol{A} \\
& =\int d s^{\prime} \frac{1-\boldsymbol{\tau}(s) \cdot \boldsymbol{\tau}\left(s^{\prime}\right)}{\left|\boldsymbol{r}_{0}(s)-\boldsymbol{r}_{0}\left(s^{\prime}\right)\right|} \lambda\left(s^{\prime}-c t_{\mathrm{ret}}\right) .
\end{aligned}
$$

The integrand in this expression remains finite when $s^{\prime} \rightarrow s$ because due to $|\boldsymbol{\tau}|=1$ the zero value of the denominator is canceled by the zero of the numerator.

A direct calculation of the transverse force $e\left(\boldsymbol{E}_{\perp}+\boldsymbol{\tau} \times \boldsymbol{H}\right)$ inside the beam using Eqs. (1) and (2) leads to diverging integrals. For a circular orbit this observation goes back to Ref. [4] and was also shown by direct calculations in [21]. In Ref. [17], the transverse force for a line-charge beam was derived only for the points on the orbit where $\lambda=0$ (either in front or behind the bunch), thus reaffirming the conclusion that this force diverges inside the beam where $\lambda \neq 0$. Nevertheless, in one of the first papers on the subject [6], the following formula for the steady-state effective horizontal force was derived:

$$
F_{x}=-2 e \kappa \lambda
$$

where $\kappa^{-1}$ is the bending radius of the orbit and $\lambda$ is the charge density per unit length at the location of the particle. The minus sign in this equation indicates that this force is centripetal, that is directed toward the center of the circular orbit. In the recent paper [22], the authors obtained an equation for an effective transverse force similar to Eq. (4) but with the factor of 2 in Eq. (4) replaced by some factor $\Lambda$, where $2 \leq \Lambda \leq 4$.

An important insight into the problem of the transverse force comes from the Hamiltonian formulation of the equations of motion of the beam particles which includes the self-field of the beam. Such analysis has been carried in Refs. [23,24] and for completeness is reproduced in Appendix A. As is well known, the particle motion in an accelerator is formulated in the standard coordinate system $x, y, s$ defined in the vicinity of the reference orbit $\boldsymbol{r}_{0}(s)$ (which we assume to be planar),

$$
\boldsymbol{r}(s)=\boldsymbol{r}_{0}(s)+\boldsymbol{n}(s) x+\boldsymbol{y} y,
$$


where $\boldsymbol{y}$ is the unit vector in the vertical direction and $\boldsymbol{n}(s)$ is the normal vector in the plane of the orbit. The unit vectors $\boldsymbol{n}(s)$ and $\boldsymbol{\tau}(s)$ satisfy the Frenet-Serret equations, ${ }^{2}$

$$
\frac{d \boldsymbol{\tau}}{d s}=-\kappa n, \quad \frac{d \boldsymbol{n}}{d s}=\kappa \boldsymbol{\tau},
$$

where $\kappa(s)$ is the curvature of the orbit defined by $\kappa=-\boldsymbol{n} \cdot d \boldsymbol{\tau} / d s$. In the Hamiltonian formalism, one considers the coordinate $s$ as a time-like variable, and the first two conjugate variables are the time $t$ and the negative Hamiltonian $-H=-\left(\gamma m c^{2}+e \phi\right)$; both are considered as functions of $s$. For our purposes, it is more convenient to use the difference $\Delta H=H-\gamma_{0} m c^{2}$ instead of $H$, where $\gamma_{0} m c^{2}$ is the nominal energy corresponding to the reference trajectory $\boldsymbol{r}_{0}(s)$. The time derivative $d t / d s$ in our ultrarelativistic limit reduces to $d t / d s=c^{-1}$. The $s$-derivative of $\Delta H$ follows directly from the energy conservation equation (an of course is derivable from the Hamiltonian formalism, see Appendix A),

$$
\frac{d \Delta H}{d s}=e \boldsymbol{\tau} \cdot \boldsymbol{E}+e \frac{d \phi}{d s}=\frac{e}{c}\left(\frac{\partial \phi}{\partial t}-\boldsymbol{\tau} \cdot \frac{\partial \boldsymbol{A}}{\partial t}\right),
$$

where in the last step we have expressed the electric field through the derivatives of the scalar and vector potentials. The right-hand side of this equation can be expressed in terms of the function $V$ given by Eq. (3),

$$
\frac{d \Delta H}{d s}=\frac{e}{c} \frac{\partial V}{\partial t}=-e \int d s^{\prime} \frac{1-\boldsymbol{\tau}(s) \cdot \boldsymbol{\tau}\left(s^{\prime}\right)}{\left|\boldsymbol{r}_{0}(s)-\boldsymbol{r}_{0}\left(s^{\prime}\right)\right|} \lambda^{\prime}\left(s^{\prime}-c t_{\mathrm{ret}}\right),
$$

where $\lambda^{\prime}$ denotes the derivative of $\lambda$ with respect to its argument.

For the equation of motion in the horizontal plane one finds (see Eq. (A19) and (A20) in Appendix A),

$$
\frac{d^{2} x}{d s^{2}}=-\kappa^{2} x+\kappa \frac{\Delta H-e \phi}{\gamma m c^{2}}+\frac{e}{\gamma m c^{2}}\left(E_{x}-B_{y}\right) .
$$

In this equation $\kappa(s)=p_{0} c / e B_{y}^{(\text {ext })}(s)$ is the local curvature of the orbit caused by the external vertical magnetic field $B_{y}^{(\mathrm{ext})}(s)$ and $p_{0}$ is the nominal momentum. ${ }^{3}$ The last term on the right-hand side of this equation is due to the transverse component of the Lorentz force. The problem with this equation is that $\phi, E_{x}$ and $B_{y}$ all diverge on the beam orbit if one uses the 1D model with Eq. (1) and (2) for the potentials and fields. However, if we combine the last

\footnotetext{
${ }^{2}$ The signs in these equations are opposite to the ones used in our earlier paper [20].

${ }^{3}$ In this equation we ignore the quadrupole and solenoidal components of the magnetic field on the orbit, which can be easily added to the right-hand side.
}

two terms in Eq. (9) with the potential $\phi$ into the renormalized force $\mathcal{F}$ in $x$-direction,

$$
\mathcal{F}=e\left(-\kappa \phi+E_{x}-B_{y}\right),
$$

then, as shown in Appendix B, this force is finite everywhere on the orbit of the beam and the general expression for the force for arbitrary curve $\boldsymbol{r}_{0}(s)$ is given by Eq. (B10). Equation (9) is then written as

$$
\frac{d^{2} x}{d s^{2}}=-\kappa^{2} x+\kappa \frac{\Delta H}{\gamma m c^{2}}+\frac{\mathcal{F}}{\gamma m c^{2}},
$$

and together with Eq. (8) it defines the 1D model which includes both the longitudinal CSR wake [as the right-hand side of Eq. (8)] and the transverse force $\mathcal{F}$. One can also show [25] that in the case of the steady-state beam moving on a circular orbit the force given by Eq. (10) reduces to Eq. (4). This our result disagrees with Ref. [22] where, as was mentioned above, the factor 2 in Eq. (4) was replaced by a factor $\Lambda \geq 2$.

At this point it seems that we have succeeded in building a 1D model that incorporates in it the effects of the transverse force, and thus affirmatively answered the question formulated at the beginning of this section. The problem however arises when we try to formulate the initial conditions for the equations of motion (8) and (11). To illustrate this problem, we will consider a passage of a relativistic beam through a bending magnet as shown in Fig. 1 (the problem first studied in Ref. [11] for the longitudinal CSR wake field). The initial conditions are formulated at the entrance to the magnet, $s=0$, after the beam has traveled along the straight line from $s=-\infty$. While the initial values of $x$ and $x^{\prime}$ for Eq. (11) are naturally taken from the beam distribution at $s=0$, the initial $\Delta H$ is equal to $\Delta H=\Delta \mathcal{E}+e \phi$, where $\Delta \mathcal{E}$ is the energy deviation from the nominal value. The potential $\phi$ at $s=0$ for a thin beam, however, as can be shown, involves $\ln \sigma_{\perp}$ and hence diverges in the limit $\sigma_{\perp} \rightarrow 0 .{ }^{4}$ In addition, one can show that the integral on the right-hand side of Eq. (8) and the integrals in Eq. (B10) also diverge at the lower limit $s=-\infty$. We come to the conclusion that even though we have equations of motion for the particles of the line charge beam, they cannot be used even in the simplest problem of a beam passing through a single bending magnet.

This difficulty forces us to reformulate the equations of motion in terms of the energy deviation of the particle $\Delta \mathcal{E}=\Delta \gamma m c^{2}$,

$$
\frac{d \Delta \mathcal{E}}{d s}=e \boldsymbol{\tau} \cdot \boldsymbol{E}
$$

\footnotetext{
${ }^{4}$ This is the reason why this potential cannot be calculated inside the beam using Eq. (1a).
} 


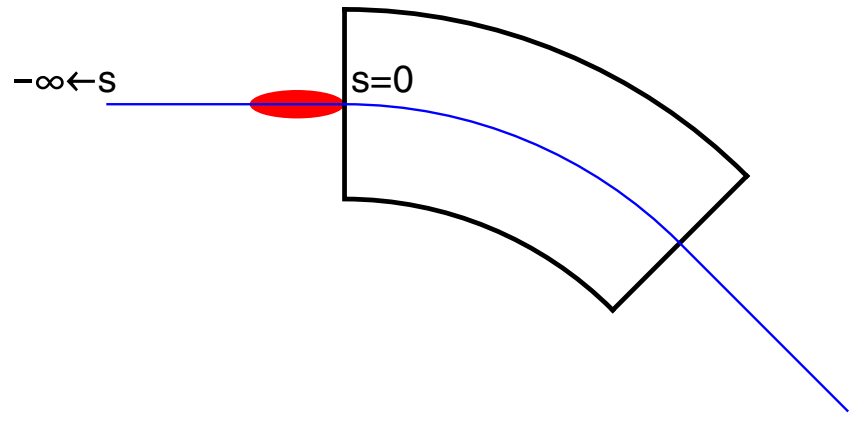

FIG. 1. A bending magnet with the beam nominal trajectory shown by the blue line. The beam shown by red is at its initial position at the entrance to the magnet.

As was proved in Ref. [12] the longitudinal part of the electric field, $\boldsymbol{\tau} \cdot \boldsymbol{E}$, of a line-charge beam is finite at the location of the bunch, so the right-hand side of Eq. (12) is well defined. In the horizontal plane, Eq. (9) is now written as

$$
\frac{d^{2} x}{d s^{2}}=-\kappa^{2} x+\kappa \frac{\Delta \mathcal{E}}{\gamma m c^{2}}+\frac{e}{\gamma m c^{2}}\left(E_{x}-B_{y}\right) .
$$

The last term in this equation is the transverse force which should be calculated with account of the finite transverse beam size $\sigma_{\perp}$.

In the next section we will derive a general expression for this transverse force.

\section{CALCULATION OF THE TRANSVERSE FORCE}

We start from the 3D retarded potentials [26] - the expressions for the scalar potential $\phi(\boldsymbol{r}, t)$ and the vector potential $\boldsymbol{A}(\boldsymbol{r}, t)$ generated by a beam with the charge density $\rho(\boldsymbol{r}, t)$ and current density $\boldsymbol{j}(\boldsymbol{r}, t)=c \boldsymbol{\beta}(\boldsymbol{r}, t) \rho(\boldsymbol{r}, t)$,

$$
\begin{aligned}
\phi(\boldsymbol{r}, t) & =\int d^{3} r^{\prime} \frac{\rho\left(\boldsymbol{r}^{\prime}, t_{\mathrm{ret}}\right)}{\left|\boldsymbol{r}^{\prime}-\boldsymbol{r}\right|}, \\
\boldsymbol{A}(\boldsymbol{r}, t), & =\int d^{3} r^{\prime} \frac{\boldsymbol{\beta}\left(\boldsymbol{r}^{\prime}, t_{\mathrm{ret}}\right) \rho\left(\boldsymbol{r}^{\prime}, t_{\mathrm{ret}}\right)}{\left|\boldsymbol{r}^{\prime}-\boldsymbol{r}\right|},
\end{aligned}
$$

where the retarded time is $t_{\text {ret }}\left(\boldsymbol{r}, \boldsymbol{r}^{\prime}, t\right)=t-\left|\boldsymbol{r}^{\prime}-\boldsymbol{r}\right| / c$ and the integration goes over the whole space. To simplify the notation, in what follows we drop the arguments $\left(\boldsymbol{r}^{\prime}, t_{\text {ret }}\left(\boldsymbol{r}, \boldsymbol{r}^{\prime}, t\right)\right)$ of the function $\rho$ and use the abbreviated notation: $\boldsymbol{\beta}\left(\boldsymbol{r}, t_{\mathrm{ret}}\left(\boldsymbol{r}, \boldsymbol{r}^{\prime}, t\right)\right) \rightarrow \boldsymbol{\beta}$ and $\boldsymbol{\beta}\left(\boldsymbol{r}^{\prime}, t_{\mathrm{ret}}\left(\boldsymbol{r}, \boldsymbol{r}^{\prime}, t\right)\right) \rightarrow \boldsymbol{\beta}^{\prime}$. The expression for the transverse part of the Lorentz force (per unit charge) is

$\frac{1}{e} \boldsymbol{F}_{\perp}=\boldsymbol{E}_{\perp}+\boldsymbol{\beta} \times \boldsymbol{B}=-\nabla_{\perp} \phi-\left.\frac{1}{c} \frac{\partial \boldsymbol{A}}{\partial t}\right|_{\perp}+\boldsymbol{\beta} \times \nabla \times \boldsymbol{A}$.
Note that the operator that selects a perpendicular to the velocity component of a vector $\boldsymbol{a}$ is

$$
\boldsymbol{a}_{\perp}=\boldsymbol{a}-\beta^{-2} \boldsymbol{\beta}(\boldsymbol{\beta} \cdot \boldsymbol{a}) .
$$

To find the contribution to $\boldsymbol{E}$ from the scalar potential, we evaluate

$$
\begin{aligned}
\nabla \phi & =\int d^{3} r^{\prime}\left(\frac{\nabla_{r} \rho}{\left|\boldsymbol{r}^{\prime}-\boldsymbol{r}\right|}+\rho \nabla_{\boldsymbol{r}} \frac{1}{\left|\boldsymbol{r}^{\prime}-\boldsymbol{r}\right|}\right) \\
& =\int d^{3} r^{\prime}\left(\frac{\nabla_{\boldsymbol{r}} \rho}{\left|\boldsymbol{r}^{\prime}-\boldsymbol{r}\right|}-\rho \frac{\boldsymbol{r}-\boldsymbol{r}^{\prime}}{\left|\boldsymbol{r}^{\prime}-\boldsymbol{r}\right|^{3}}\right) .
\end{aligned}
$$

The second term in this expression diverges in $2 \mathrm{D}$, but converges in 3D. As we will see below this singularity will be partially canceled by another term in the Lorentz force, leading to the convergence of the total force in 2D as well. For the contribution to $\boldsymbol{E}$ from the vector potential we need to calculate the time derivative of $\boldsymbol{A}$,

$$
\frac{\partial \boldsymbol{A}}{\partial t}=\int \frac{d^{3} r^{\prime}}{\left|\boldsymbol{r}^{\prime}-\boldsymbol{r}\right|}\left(\boldsymbol{\beta}^{\prime} \partial_{t_{\mathrm{rel}}} \rho+\rho \partial_{t_{\mathrm{ret}}} \boldsymbol{\beta}^{\prime}\right)
$$

where we have used the fact that $\partial_{t}=\partial_{t_{\text {ret }}}$. We also need to calculate $\nabla \times \boldsymbol{A}$,

$$
\begin{aligned}
\nabla \times \boldsymbol{A}(\boldsymbol{r}, t)= & \int d^{3} r^{\prime}\left[\rho\left(\nabla_{\boldsymbol{r}} \frac{1}{\left|\boldsymbol{r}^{\prime}-\boldsymbol{r}\right|}\right) \times \boldsymbol{\beta}^{\prime}\right. \\
& \left.+\frac{1}{\left|\boldsymbol{r}^{\prime}-\boldsymbol{r}\right|}\left(\nabla_{\boldsymbol{r}} \rho\right) \times \boldsymbol{\beta}^{\prime}+\frac{1}{\left|\boldsymbol{r}^{\prime}-\boldsymbol{r}\right|} \rho \nabla_{\boldsymbol{r}} \times \boldsymbol{\beta}^{\prime}\right] \\
= & \int d^{3} r^{\prime}\left[-\rho \frac{\boldsymbol{r}-\boldsymbol{r}^{\prime}}{\left|\boldsymbol{r}^{\prime}-\boldsymbol{r}\right|^{3}} \times \boldsymbol{\beta}^{\prime}+\frac{1}{\left|\boldsymbol{r}^{\prime}-\boldsymbol{r}\right|}\left(\nabla_{\boldsymbol{r}} \rho\right) \times \boldsymbol{\beta}^{\prime}\right. \\
& \left.+\frac{1}{\left|\boldsymbol{r}^{\prime}-\boldsymbol{r}\right|} \rho \nabla_{\boldsymbol{r}} \times \boldsymbol{\beta}^{\prime}\right]
\end{aligned}
$$

which gives for the double vectorial product $\boldsymbol{\beta} \times \nabla \times \boldsymbol{A}$,

$$
\begin{aligned}
\boldsymbol{\beta} \times \nabla \times \boldsymbol{A}= & -\int \frac{d^{3} r^{\prime} \rho}{\left|\boldsymbol{r}^{\prime}-\boldsymbol{r}\right|^{3}} \boldsymbol{\beta} \times\left(\boldsymbol{r}-\boldsymbol{r}^{\prime}\right) \times \boldsymbol{\beta}^{\prime} \\
& +\int \frac{d^{3} r^{\prime}}{\left|\boldsymbol{r}^{\prime}-\boldsymbol{r}\right|}\left[\boldsymbol{\beta} \times\left(\nabla_{\boldsymbol{r}} \rho\right) \times \boldsymbol{\beta}^{\prime}+\rho \boldsymbol{\beta} \times \nabla_{\boldsymbol{r}} \times \boldsymbol{\beta}^{\prime}\right] .
\end{aligned}
$$

Up to this point we did not make assumptions regarding the value of $\beta$. In what follows we will assume $\beta=1$. For the transverse component of $-\nabla \phi$ we then have,

$$
\begin{aligned}
-\nabla_{\perp} \phi= & -\int \frac{d^{3} r^{\prime}}{\left|\boldsymbol{r}^{\prime}-\boldsymbol{r}\right|}\left[\nabla_{\boldsymbol{r}} \rho-\boldsymbol{\beta}\left(\boldsymbol{\beta} \cdot \nabla_{\boldsymbol{r}} \rho\right)\right] \\
& +\int \frac{d^{3} r^{\prime} \rho}{\left|\boldsymbol{r}^{\prime}-\boldsymbol{r}\right|^{3}}\left[\left(\boldsymbol{r}-\boldsymbol{r}^{\prime}\right)-\boldsymbol{\beta}\left(\boldsymbol{\beta} \cdot\left(\boldsymbol{r}-\boldsymbol{r}^{\prime}\right)\right)\right] .
\end{aligned}
$$


Similarly, for the transverse component of the time derivative of the vector potential we obtain

$$
\begin{aligned}
-\left.\frac{1}{c} \frac{\partial \boldsymbol{A}}{\partial t}\right|_{\perp}= & -\frac{1}{c} \int \frac{d^{3} r^{\prime}}{\left|\boldsymbol{r}^{\prime}-\boldsymbol{r}\right|}\left[\boldsymbol{\beta}^{\prime}-\boldsymbol{\beta}\left(\boldsymbol{\beta}^{\prime} \cdot \boldsymbol{\beta}\right)\right] \partial_{t_{\mathrm{ret}}} \rho \\
& -\frac{1}{c} \int \frac{d^{3} r^{\prime}}{\left|\boldsymbol{r}^{\prime}-\boldsymbol{r}\right|} \rho\left[\partial_{t_{\mathrm{ret}}} \boldsymbol{\beta}^{\prime}-\boldsymbol{\beta}\left(\boldsymbol{\beta} \cdot \partial_{t_{\mathrm{ret}}} \boldsymbol{\beta}^{\prime}\right)\right] .
\end{aligned}
$$

We now combine all terms into the transverse force (15). Using the expressions

$$
\nabla_{\boldsymbol{r}} \times \boldsymbol{\beta}^{\prime}=-\frac{1}{c} \frac{1}{\left|\boldsymbol{r}^{\prime}-\boldsymbol{r}\right|}\left(\boldsymbol{r}-\boldsymbol{r}^{\prime}\right) \times\left(\partial_{\mathrm{trel}_{\mathrm{rel}}} \boldsymbol{\beta}^{\prime}\right)
$$

and

$$
\nabla_{r} \rho=-\frac{1}{c} \partial_{t_{\mathrm{ret}}} \rho \frac{\boldsymbol{r}-\boldsymbol{r}^{\prime}}{\left|\boldsymbol{r}-\boldsymbol{r}^{\prime}\right|}
$$

after some transformations we arrive at the following result

$$
\begin{aligned}
& \frac{1}{e} \boldsymbol{F}_{\perp}=\int \frac{d^{3} r^{\prime} \rho}{\left|\boldsymbol{r}^{\prime}-\boldsymbol{r}\right|^{3}}\left[\left(\boldsymbol{r}-\boldsymbol{r}^{\prime}\right)\left(1-\boldsymbol{\beta} \cdot \boldsymbol{\beta}^{\prime}\right)+\left(\boldsymbol{\beta}^{\prime}-\boldsymbol{\beta}\right)\left(\boldsymbol{\beta} \cdot\left(\boldsymbol{r}-\boldsymbol{r}^{\prime}\right)\right)\right]+\frac{1}{c} \int \frac{d^{3} r^{\prime} \partial_{t_{\mathrm{ret}}} \rho}{\left|\boldsymbol{r}^{\prime}-\boldsymbol{r}\right|^{2}}\left[\left(\boldsymbol{r}-\boldsymbol{r}^{\prime}\right)\left(1-\boldsymbol{\beta} \cdot \boldsymbol{\beta}^{\prime}\right)+\left(\boldsymbol{\beta}^{\prime}-\boldsymbol{\beta}\right)\left(\boldsymbol{\beta} \cdot\left(\boldsymbol{r}-\boldsymbol{r}^{\prime}\right)\right)\right] \\
& -\frac{1}{c} \int \frac{d^{3} r^{\prime} \partial_{t_{\mathrm{ret}}} \rho}{\left|\boldsymbol{r}^{\prime}-\boldsymbol{r}\right|}\left[\boldsymbol{\beta}^{\prime}-\boldsymbol{\beta}\left(\boldsymbol{\beta} \cdot \boldsymbol{\beta}^{\prime}\right)\right]-\frac{1}{c} \int \frac{d^{3} r^{\prime} \rho}{\left|\boldsymbol{r}^{\prime}-\boldsymbol{r}\right|^{2}} \boldsymbol{\beta} \times\left(\boldsymbol{r}-\boldsymbol{r}^{\prime}\right) \times\left(\partial_{\mathrm{trel}_{\mathrm{ret}}} \boldsymbol{\beta}^{\prime}\right)-\frac{1}{c} \int \frac{d^{3} r^{\prime} \rho}{\left|\boldsymbol{r}^{\prime}-\boldsymbol{r}\right|}\left[\partial_{\mathrm{trel}} \boldsymbol{\beta}^{\prime}-\boldsymbol{\beta}\left(\boldsymbol{\beta} \cdot \partial_{t_{\mathrm{rel}}} \boldsymbol{\beta}^{\prime}\right)\right] .
\end{aligned}
$$

The time derivative $\partial_{t_{\text {rel }}} \boldsymbol{\beta}^{\prime}$ in Eq. (23) is due to a possible variation of the transverse component of the local velocity within the beam. This component is typically small, and in a good approximation can be ignored by setting

$$
\boldsymbol{\beta}(x, y, s, t)=\boldsymbol{\tau}(s) .
$$

Then $\partial_{t_{\mathrm{re}}} \boldsymbol{\beta}^{\prime}$ vanishes together with the last two terms in Eq. (23). Projecting the transverse force onto the normal vector $\boldsymbol{n}(s), F_{\perp}=\boldsymbol{n} \cdot \boldsymbol{F}$, we obtain,

$$
\begin{aligned}
\frac{1}{e} F_{\perp}= & \int \frac{d^{3} r^{\prime} \rho}{\left|\boldsymbol{r}^{\prime}-\boldsymbol{r}\right|^{3}}\left(\boldsymbol{r}-\boldsymbol{r}^{\prime}\right) \cdot\left[\boldsymbol{n}\left(1-\boldsymbol{\tau} \cdot \boldsymbol{\tau}^{\prime}\right)+\boldsymbol{\tau}\left(\boldsymbol{n} \cdot \boldsymbol{\tau}^{\prime}\right)\right] \\
& +\frac{1}{c} \int \frac{d^{3} r^{\prime} \partial_{t_{\mathrm{ret}}} \rho}{\left|\boldsymbol{r}^{\prime}-\boldsymbol{r}\right|^{2}}\left(\boldsymbol{r}-\boldsymbol{r}^{\prime}\right) \cdot\left[\boldsymbol{n}\left(1-\boldsymbol{\tau} \cdot \boldsymbol{\tau}^{\prime}\right)+\boldsymbol{\tau}\left(\boldsymbol{n} \cdot \boldsymbol{\tau}^{\prime}\right)\right] \\
& -\frac{1}{c} \int \frac{d^{3} r^{\prime} \partial_{t_{\mathrm{ret}}} \rho}{\left|\boldsymbol{r}^{\prime}-\boldsymbol{r}\right|} \boldsymbol{n} \cdot \boldsymbol{\tau}^{\prime},
\end{aligned}
$$

In this equation we continue to use the abbreviated notation introduced in the previous section expanding it with $\boldsymbol{\tau}\left(s^{\prime}\right) \rightarrow \boldsymbol{\tau}^{\prime}, \boldsymbol{n}\left(s^{\prime}\right) \rightarrow \boldsymbol{n}^{\prime}$. Equation (25) can be further simplified if we note that

$$
\boldsymbol{\tau}\left(\boldsymbol{n} \cdot \boldsymbol{\tau}^{\prime}\right)-\boldsymbol{n}\left(\boldsymbol{\tau} \cdot \boldsymbol{\tau}^{\prime}\right)=-\boldsymbol{n}^{\prime}
$$

Indeed the left-hand side of this equation is orthogonal to $\boldsymbol{\tau}^{\prime}$ which is verified by taking the dot product. The minus sign on the right-hand side is found if we take the limit $\boldsymbol{n}^{\prime} \rightarrow \boldsymbol{n}$ and $\boldsymbol{\tau}^{\prime} \rightarrow \boldsymbol{\tau}$. Using Eq. (26) we obtain

$$
\begin{aligned}
\frac{1}{e} F_{\perp}= & \frac{1}{c} \int \frac{d^{3} r^{\prime} \partial_{t_{\mathrm{ret}}} \rho}{\left|\boldsymbol{r}^{\prime}-\boldsymbol{r}\right|^{2}}\left[\left(\boldsymbol{r}-\boldsymbol{r}^{\prime}\right) \cdot\left(\boldsymbol{n}-\boldsymbol{n}^{\prime}\right)-\left|\boldsymbol{r}^{\prime}-\boldsymbol{r}\right|\left(\boldsymbol{n} \cdot \boldsymbol{\tau}^{\prime}\right)\right] \\
& +\int \frac{d^{3} r^{\prime} \rho}{\left|\boldsymbol{r}^{\prime}-\boldsymbol{r}\right|^{3}}\left(\boldsymbol{r}-\boldsymbol{r}^{\prime}\right) \cdot\left(\boldsymbol{n}-\boldsymbol{n}^{\prime}\right)
\end{aligned}
$$

Analysis shows that these integrals converge not only in $3 \mathrm{D}$, but also in $2 \mathrm{D}$, that is for a beam in which the vertical dimension is negligibly small and the integration goes in the plane of the orbit, $d^{3} r^{\prime} \rightarrow d^{2} r^{\prime}$.

Note that for a plane orbit that we assume here the transverse force (25) lies in the plane of the orbit ( $x$-direction). There is also a component of the transverse force in the $y$-direction which is relatively small when compared with $F_{\perp}$; we ignore this part of the force in what follows.

\section{STEADY-STATE TRANSVERSE FORCE ON A CIRCULAR ORBIT}

We will now consider a circular orbit of constant radius $\kappa^{-1}$ and will take into account the small size of the beam assuming that $\sigma_{z}, \sigma_{\perp} \ll \kappa^{-1}$. In the standard curvilinear coordinate system $x, y, s$, related to the reference orbit $\boldsymbol{r}_{0}(s)$ (see details in Appendix A), the 3D integration in Eq. (27) reduces to, ${ }^{5}$

$$
\int d^{3} r^{\prime} \rightarrow \int d x^{\prime} d s^{\prime} d y^{\prime}
$$

Our derivation is considerably simplified if we also assume a long-thin beam, $\sigma_{\perp} \ll \sigma_{z}$. This requirement turns out to be not crucial and we will further discuss its necessity

\footnotetext{
${ }^{5}$ In a more precise expression for the integral, $d x$ should be replaced by $d x /(1+\kappa x)$, but this leads to a negligibly small correction to our result because we assume $\sigma_{\perp} \ll \kappa^{-1}$.
} 
in the next section. For a long-thin beam, the first integral in Eq. (27), which we will denote by $I_{1}$, can be reduced to a 1D integral if we carry out the integration over $x, y$ coordinates and use the 1D distribution $\lambda=$ $\int d x^{\prime} d y^{\prime} \rho\left(\boldsymbol{r}^{\prime}, t_{\text {ret }}\right)$,

$$
\begin{aligned}
I_{1} & \equiv \frac{1}{c} \int \frac{d x^{\prime} d y^{\prime} d s^{\prime} \partial_{t_{\mathrm{ret}}} \rho}{\left|\boldsymbol{r}^{\prime}-\boldsymbol{r}\right|^{2}}\left[\left(\boldsymbol{r}-\boldsymbol{r}^{\prime}\right) \cdot\left(\boldsymbol{n}-\boldsymbol{n}^{\prime}\right)-\left|\boldsymbol{r}^{\prime}-\boldsymbol{r}\right|\left(\boldsymbol{n} \cdot \boldsymbol{\tau}^{\prime}\right)\right] \\
& \rightarrow-\int \frac{d s^{\prime} \lambda^{\prime}}{\left|\boldsymbol{r}_{0}^{\prime}-\boldsymbol{r}_{0}\right|^{2}}\left[\left(\boldsymbol{r}_{0}-\boldsymbol{r}_{0}^{\prime}\right) \cdot\left(\boldsymbol{n}-\boldsymbol{n}^{\prime}\right)-\left(\boldsymbol{n} \cdot \boldsymbol{\tau}^{\prime}\right)\left|\boldsymbol{r}_{0}^{\prime}-\boldsymbol{r}_{0}\right|\right],
\end{aligned}
$$

where we took into account that $\lambda$ is a function of $s^{\prime}-c t_{\text {ret }}$ so that $\partial_{t_{\text {ret }}} \lambda\left(s^{\prime}-c t_{\text {ret }}\right)=-c \lambda^{\prime}\left(s^{\prime}-c t_{\text {ret }}\right) \rightarrow-c \lambda^{\prime}$, with $\lambda^{\prime}$ the derivative of the function $\lambda$ with respect to its argument. We also use the abbreviated notations: $\boldsymbol{r}_{0}(s) \rightarrow \boldsymbol{r}_{0}, \boldsymbol{r}_{0}\left(s^{\prime}\right) \rightarrow \boldsymbol{r}_{0}^{\prime}$.

Due to the smallness of the beam, the path length $s^{\prime}-s$ involved in the integral of Eq. (29) is much smaller than the orbit radius $\kappa^{-1}$ and we can use the following approximations,

$$
\begin{aligned}
\left|\boldsymbol{r}_{0}^{\prime}-\boldsymbol{r}_{0}\right| & \approx\left|s-s^{\prime}\right|, \\
\left(\boldsymbol{r}_{0}-\boldsymbol{r}_{0}^{\prime}\right) \cdot\left(\boldsymbol{n}-\boldsymbol{n}^{\prime}\right) & \approx \kappa\left(s-s^{\prime}\right)^{2}, \\
\boldsymbol{n} \cdot \boldsymbol{\tau}^{\prime} & \approx \kappa\left(s-s^{\prime}\right) .
\end{aligned}
$$

The argument $s^{\prime}-c t_{\text {ret }}$ of $\lambda^{\prime}$ in Eq. (29) can also be approximated: for $s^{\prime}<s$,

$$
\begin{aligned}
s^{\prime}-c t_{\mathrm{ret}} & =s^{\prime}-c t+2 \kappa^{-1} \sin \left(\kappa \frac{s-s^{\prime}}{2}\right) \\
& \approx u-\frac{1}{24} \kappa^{2}\left(s-s^{\prime}\right)^{3}
\end{aligned}
$$

where $u=s-c t$; and for $s^{\prime}>s$,

$$
\begin{aligned}
s^{\prime}-c t_{\mathrm{ret}} & =s^{\prime}-c t+2 \kappa^{-1} \sin \left(\kappa \frac{s^{\prime}-s}{2}\right) \\
& \approx u+2\left(s^{\prime}-s\right) .
\end{aligned}
$$

The following approximation is also valid,

$$
\frac{\boldsymbol{n} \cdot \boldsymbol{\tau}^{\prime}}{\left|\boldsymbol{r}_{0}^{\prime}-\boldsymbol{r}_{0}\right|} \approx \kappa\left[1-\frac{1}{8} \kappa^{2}\left(s-s^{\prime}\right)^{2}\right] \operatorname{sgn}\left(s-s^{\prime}\right) .
$$

Substituting the above expressions into Eq. (29) we obtain,

$$
\begin{aligned}
& -\int \frac{d s^{\prime} \lambda^{\prime}\left(s^{\prime}-c t_{\mathrm{ret}}\right)}{\left|\boldsymbol{r}_{0}^{\prime}-\boldsymbol{r}_{0}\right|^{2}}\left[\left(\boldsymbol{r}_{0}-\boldsymbol{r}_{0}^{\prime}\right) \cdot\left(\boldsymbol{n}-\boldsymbol{n}^{\prime}\right)-\boldsymbol{n} \cdot \boldsymbol{\tau}^{\prime}\left|\boldsymbol{r}_{0}^{\prime}-\boldsymbol{r}_{0}\right|\right] \\
& \quad=\kappa \int_{-\infty}^{\infty} d s^{\prime} \lambda^{\prime}\left(s^{\prime}-c t_{\mathrm{ret}}\right)\left\{-1+\left[1-\frac{1}{8} \kappa^{2}\left(s-s^{\prime}\right)^{2}\right] \operatorname{sgn}\left(s-s^{\prime}\right)\right\} \\
& \quad \approx-\frac{1}{8} \kappa^{3} \int_{-\infty}^{s} d s^{\prime}\left(s-s^{\prime}\right)^{2} \lambda^{\prime}\left[u-\frac{1}{24} \kappa^{2}\left(s-s^{\prime}\right)^{3}\right]-2 \kappa \int_{s}^{\infty} d s^{\prime} \lambda^{\prime}\left(u+2\left(s^{\prime}-s\right)\right)=0 .
\end{aligned}
$$

We see that for a circular orbit this integral vanishes.

We now consider the second integral in Eq. (27) which we will denote by $I_{2}$. It is easy to see that this integral cannot be taken in the 1D approximation as with did above, because it would logarithmically diverge at $s^{\prime}=s$. To overcome this problem, we split the integration region into three intervals. In the first one we integrate over $s^{\prime}$ from $-\infty$ to $s-\Delta s$, in the second one we integrate from $s-\Delta s$ to $s+\Delta s$, and in the third interval we integrate from $s+\Delta s$ to $\infty$. We choose $\Delta s$ such that $\sigma_{z} \gg \Delta s \gg \sigma_{\perp}$ (here we use our assumption $\sigma_{z} \gg \sigma_{\perp}$ ). The integration over the first and the third intervals can be carried out in the linecharge approximation, as we did in $I_{1}$, using Eqs. (30), (31), and (32). In the first interval, which we denote $I_{2,1}$, we have

$$
\begin{aligned}
I_{2,1} \approx & \kappa \int_{-\infty}^{s-\Delta s} \frac{d s^{\prime}}{s-s^{\prime}} \lambda\left(u-\frac{1}{24} \kappa^{2}\left(s-s^{\prime}\right)^{3}\right) \\
\approx & -\frac{1}{3} \kappa \lambda(u) \ln \left[(1 / 24) \kappa^{2} \Delta s^{3}\right] \\
& +\frac{1}{3} \kappa \int_{0}^{\infty} \ln (\zeta) \lambda^{\prime}(u-\zeta) d \zeta,
\end{aligned}
$$

where we have introduced the new integration variable $\zeta=(1 / 24) \kappa^{2}\left(s-s^{\prime}\right)^{3}$ and integrated by parts to arrive at the result; we also neglected small terms in the final expression. Similarly, we use the line-charge approximation to calculate the third integral, $I_{2,3}$, 


$$
\begin{aligned}
I_{2,3} & \approx \kappa \int_{s+\Delta s}^{\infty} \frac{d s^{\prime}}{s^{\prime}-s} \lambda\left(u+2\left(s^{\prime}-s\right)\right) \\
& \approx-\kappa \ln (2 \Delta s) \lambda(u)-\kappa \int_{0}^{\infty} \ln (\zeta) \lambda^{\prime}(u+\zeta) d \zeta,
\end{aligned}
$$

where the new integration variable is $\zeta=2\left(s^{\prime}-s\right)$. Combining the two integrals we obtain

$$
\begin{aligned}
I_{2,1}+I_{2,3}= & -\kappa \lambda(u)\left[\frac{1}{3} \ln \left(\kappa^{2} / 3\right)+\ln \left(\Delta s^{2}\right)\right] \\
& +\kappa \int_{0}^{\infty} \ln (\zeta)\left[\frac{1}{3} \lambda^{\prime}(u-\zeta)-\lambda^{\prime}(u+\zeta)\right] d \zeta .
\end{aligned}
$$

Turning now to the second interval, $s-\Delta s<s^{\prime}<$ $s+\Delta s$, we need to carry out a 3D integration. Because of the smallness of $\Delta s$, we can neglect the retardation term in the argument of $\rho, \rho\left(\boldsymbol{r}^{\prime}, t_{\text {ret }}\right) \rightarrow \rho\left(\boldsymbol{r}^{\prime}, t\right)$ (this approximation is valid only if $\sigma_{z} \gg \sigma_{\perp}$ which we have assumed above). Furthermore, we will also assume that in this small region the distribution function can be represented as a product of the longitudinal distribution $\lambda(u)$ and a transverse distribution $\mu(x, y), \rho(\boldsymbol{r}, t)=\lambda(u) \mu(x, y)$. Using the notation $z^{\prime}=s^{\prime}-s$ and the smallness $\left|z^{\prime}\right| \leq \Delta s \ll \kappa^{-1}$, we have $\left(\boldsymbol{r}-\boldsymbol{r}^{\prime}\right) \cdot\left(\boldsymbol{n}-\boldsymbol{n}^{\prime}\right) \approx \kappa z^{\prime 2}$, which gives for the contribution from the middle interval, $I_{2,2}$,

$I_{2,2}=\lambda(u) \kappa \int_{-\Delta s}^{\Delta s} d z^{\prime} \int d x^{\prime} d y^{\prime} \frac{z^{\prime 2} \mu\left(x^{\prime}, y^{\prime}\right)}{\left(z^{\prime 2}+\left(x^{\prime}-x\right)^{2}+\left(y^{\prime}-y\right)^{2}\right)^{3 / 2}}$.

We first carry out the integral over $z^{\prime}$ using

$$
\begin{aligned}
\int_{-\Delta s}^{\Delta s} d z^{\prime} \frac{z^{\prime 2}}{\left(z^{\prime 2}+l^{2}\right)^{3 / 2}} & =2\left[\operatorname{arcsinh}\left(\frac{\Delta s}{l}\right)-\frac{\Delta s}{\sqrt{l^{2}+\Delta s^{2}}}\right] \\
& \approx \ln \left(\frac{\Delta s^{2}}{l^{2}}\right)+2(\ln 2-1) .
\end{aligned}
$$

Here $l^{2}=\left(x^{\prime}-x\right)^{2}+\left(y^{\prime}-y\right)^{2}$ and on the last step we took into account that $\Delta s \gg l$ which follows from our choice $\Delta s \gg \sigma_{\perp}$. We then have

$I_{2,2}(u, x, y)=\lambda(u) \kappa\left[\ln \left(\frac{\Delta s^{2}}{\sigma_{\perp}^{2}}\right)+2(\ln 2-1)-G(x, y)\right]$,

where

$G(x, y)=-\int_{-\infty}^{\infty} d x^{\prime} d y^{\prime} \mu\left(x^{\prime}, y^{\prime}\right) \ln \left[\frac{\left(x-x^{\prime}\right)^{2}+\left(y-y^{\prime}\right)^{2}}{\sigma_{\perp}^{2}}\right]$.
The parameter $\sigma_{\perp}$ in Eqs. (40) and (41) is added to have a dimensionless variable in the logarithm function-the final result does not depend on the value of this parameter.

We now combine Eq. (37) with Eq. (40) to obtain the final expression for the transverse force on a circular orbit,

$$
\begin{aligned}
\frac{1}{e} F_{\perp}= & I_{2,1}+I_{2,2}+I_{2,3} \\
= & \lambda(u) \kappa\left[2 \ln \left(\frac{\sigma_{z}^{2 / 3}}{\kappa^{1 / 3} \sigma_{\perp}}\right)+2 \ln 2-2+\frac{1}{3} \ln 3+G(x, y)\right] \\
& +\kappa \int_{0}^{\infty} \ln \left(\frac{\zeta}{\sigma_{z}}\right)\left[\frac{1}{3} \lambda^{\prime}(u-\zeta)-\lambda^{\prime}(u+\zeta)\right] d \zeta
\end{aligned}
$$

As expected, the auxiliary variable $\Delta s$ disappeared from the final result.

We note that a special case of the transverse force for a beam with a uniform radial density distribution was earlier derived in Ref. [21] using a method that differs from what is employed in this paper. In Appendix $\mathrm{C}$ we compare the result of Ref. [21] with Eq. (42) and show that they agree with each other. In that sense Eq. (42) generalizes the result of Ref. [21] for the case of arbitrary transverse density distribution in the beam. One can also find in Ref. [21] an example of using the transverse force for calculation of the emittance growth of a beam after passing through a bending magnet.

\section{COMPARISON WITH NUMERICAL RESULTS AND THE TRANSIENT TRANSVERSE FORCE}

In this section we will compare our analytical results with numerically calculated profiles of the transverse force from Ref. [27] using the computer code PyCSR3D [28]. The code calculates the transverse force from first principles using an integrated Green function for the electromagnetic field of a point charge moving on a circular orbit. The calculations with PyCSR3D were carried out for two sets of parameters shown in Table I assuming Gaussian beam distributions in all directions. Because in theory we assume $\gamma=\infty$, the calculations in PyCSR3D were carried out for a large value of $\gamma=2000$ when the conventional space charge effects in the transverse force are negligible.

TABLE I. Test parameter sets for PyCSR3D calculations.

\begin{tabular}{lcccc}
\hline \hline Parameter set & $\sigma_{x}(\mu \mathrm{m})$ & $\sigma_{y}(\mu \mathrm{m})$ & $\sigma_{z}(\mu \mathrm{m})$ & $\kappa^{-1}(\mathrm{~m})$ \\
\hline $\mathrm{A}$ & 10 & 10 & 10 & 1 \\
$\mathrm{~B}$ & 10 & 1 & 10 & 1 \\
\hline \hline
\end{tabular}



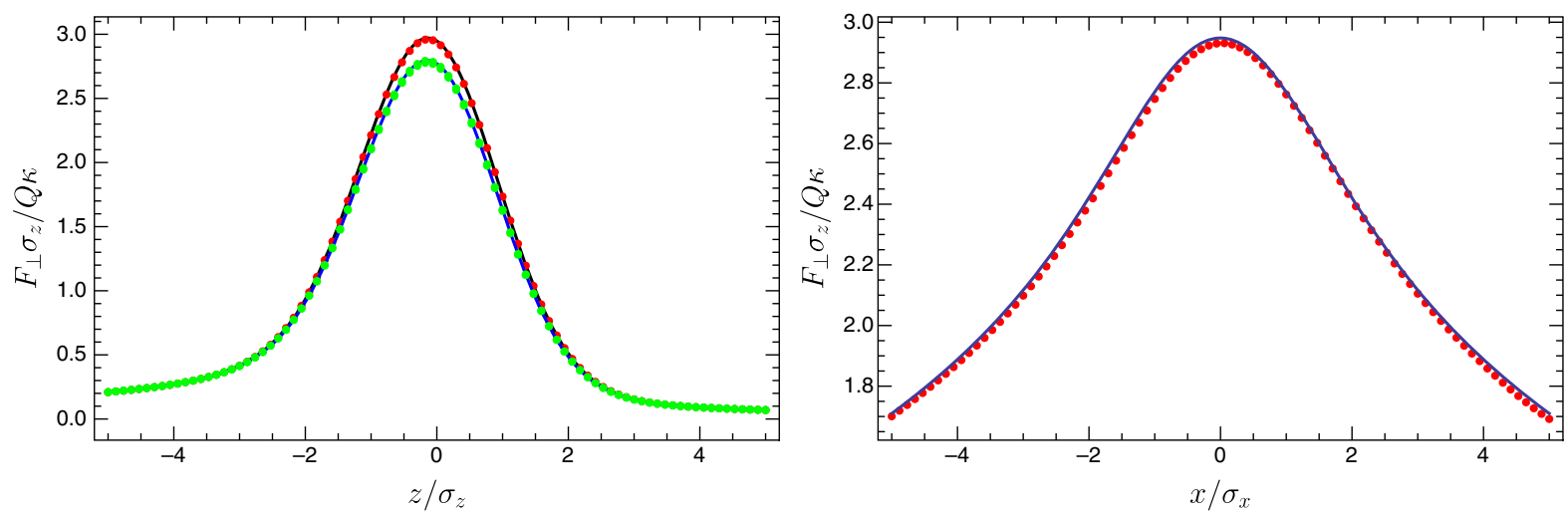

FIG. 2. Transverse force for the parameter set A. The solid black and blue lines are calculated using Eqs. (42) and (43) and the dots show the results of the numerical calculations with PyCSR3D. Left panel: dependence of $F_{\perp}$ versus $z$ for $r=0$ (on axis) and $r=\sigma_{\perp}$, respectively (the values on the axis are larger than at $r=\sigma_{\perp}$ ). Right panel: dependence of $F_{\perp}$ versus $x$ for $z=0$. The force is normalized by $Q \kappa / \sigma_{z}$ where $Q$ is the charge of the beam.

The calculated profiles of the transverse force for the parameter set A are shown in Fig. 2. Because of the axisymmetry of the distribution function in this case, $\sigma_{x}=\sigma_{y}=\sigma_{\perp}$, the function $G(x, y)$ in Eq. (42) depends only on the radial distance from the axis, $r=\sqrt{x^{2}+y^{2}}$, and is given by the following equation,

$$
\begin{aligned}
G(r) & =-\frac{1}{2 \pi \sigma_{\perp}^{2}} \int_{-\infty}^{\infty} d x^{\prime} d y^{\prime} e^{-\left(x^{\prime 2}+y^{\prime 2}\right) / 2 \sigma_{\perp}^{2}} \ln \left(\frac{\left(x-x^{\prime}\right)^{2}+\left(y-y^{\prime}\right)^{2}}{\sigma_{\perp}^{2}}\right) \\
& =-\frac{1}{2 \pi \sigma_{\perp}^{2}} \int_{0}^{\infty} r^{\prime} d r^{\prime} \int_{0}^{2 \pi} d \theta e^{-r^{\prime 2} / 2 \sigma_{\perp}^{2}} \ln \left(\frac{r^{2}+r^{\prime 2}-2 r r^{\prime} \cos \theta}{\sigma_{\perp}^{2}}\right) .
\end{aligned}
$$

The last integral is easily computed numerically.

The parameter set B calculated with PyCSR3D was compared with analytical formulas for a flat beam, that is a beam in which $\sigma_{y}=0$. In this limit, for $y=0$, the function $G$ depends only on the horizontal offset $x$ and is given by the following equation,

$G(x)=-\frac{1}{\sqrt{2 \pi} \sigma_{x}} \int_{-\infty}^{\infty} d x^{\prime} e^{-x^{\prime 2} / 2 \sigma_{x}^{2}} \ln \left(\frac{\left(x-x^{\prime}\right)^{2}}{\sigma_{x}^{2}}\right)$.

The result of the comparison is shown in Fig. 3. Note that in contrast with Fig. 2 where we find a perfect agreement, Fig. 3 shows a small discrepancy between our theory and PyCSR3D. This is explained by the fact that in theory we assume an infinitely thin beam in the vertical direction while in simulations the vertical size of the beam is finite, $\sigma_{y}=1 \mu \mathrm{m}$.

Also note that in both cases, $\mathrm{A}$ and $\mathrm{B}$, we have the horizontal beam size equal to $\sigma_{z}$ in violation of the assumption $\sigma_{\perp} \ll \sigma_{z}$ made in the derivation of Sec. IV and nevertheless find an excellent agreement between our analytical formula and the code. This can be explained as follows: the assumption $\sigma_{\perp} \ll$ $\sigma_{z}$ is only needed to simplify the derivation of Eq. (42), and not for its validity. This can also be understood by the fact that the ratio $\sigma_{\perp} / \sigma_{z}$ is not invariant under the Lorentz transformations - moving into a frame of reference that travels parallel to the beam with a relativistic velocity changes the bunch length $\sigma_{z}$ but not $\sigma_{\perp}$. Hence even if the inequality $\sigma_{\perp} \ll \sigma_{z}$ does not hold in one frame of reference it may be valid in another one.

Equation (27) is not limited to the case of the circular motion and allows to compute the transverse force at the entrance and the exit from a bending magnet of finite length. These calculations use the same simplifications as in Sec. IV wherein the two integrals in Eq. (27) are split into the parts $I_{1}, I_{2,1}, I_{2,2}$, and $I_{2,3}$ and all of them are calculated in 1D approximation except for $I_{2,2}$ for which we used Eq. (40). In the more general case of a transient wake, in contrast to the steady state result of Sec. IV, we find that $I_{1} \neq 0$, and there are contributions from the straight parts of the orbit before and after the entrance to the integrals $I_{1}, I_{2,1}$ and $I_{2,1}$. An example of calculations of the transient transverse force is shown in Figs. 4 (entrance to the bend) and 5 (exit from the bend) for the parameter set A.

It is interesting to note how fast the transverse force reaches the steady state at the entrance to the bend. For comparison, we mention here that the formation length [9] for the longitudinal CSR wake in this case is 


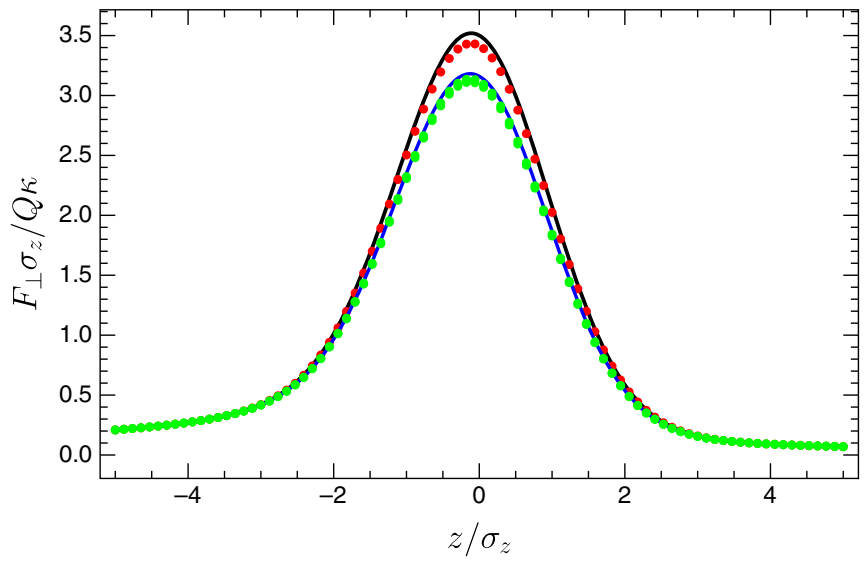

FIG. 3. Transverse force for the parameter set B. The solid black and blue lines are calculated using Eq. (44) with $x=0$ (on axis) and $x=\sigma_{x}$, respectively. The dots show the results of the numerical calculations with PyCSR3D for the same offsets. The force is normalized by $Q \kappa / \sigma_{z}$ where $Q$ is the charge of the beam.

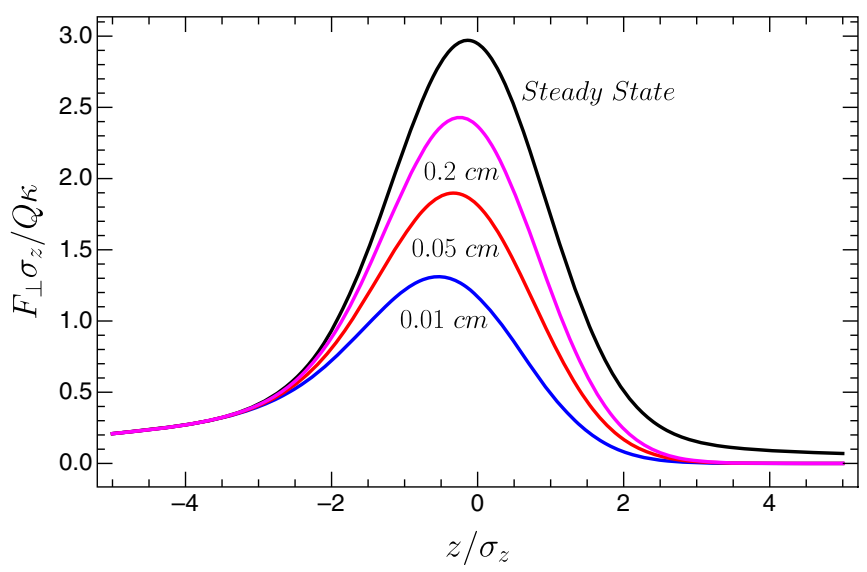

FIG. 4. Transient transverse force on the axis of the beam at the entrance to the bend. The numbers near each curve show the distance from the entrance.

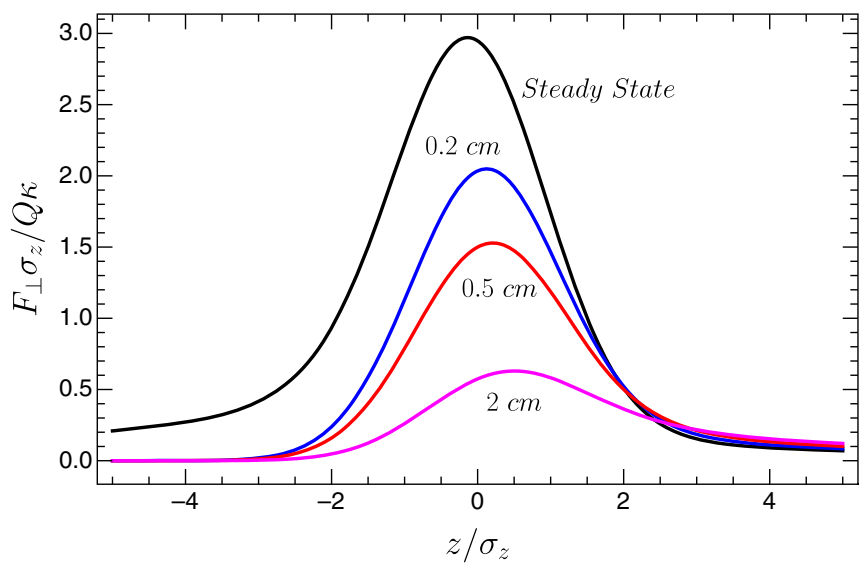

FIG. 5. Transient transverse force on the axis of the beam after the exit from the bend. The numbers near each curve show the distance from the exit. $\left(24 \sigma_{z} \kappa^{-2}\right)^{1 / 3}=6.7 \mathrm{~cm}$, while we see from Fig. 4 that the transverse force builds up on a much shorter, millimeter scale. This is an indication of the local nature of the transverse force-a large part of this force is carried out by the interaction of nearby particles and not so much by the retarded electromagnetic radiation. The decay of the transverse force in Fig. 5 at the exit from the bend is also relatively fast.

\section{SUMMARY}

In this paper, we showed that formulating the equations of motion of particles in a relativistic beam in Hamiltonian form and renormalizing the transverse force, allows one to calculate this force in 1D, or line-charge, approximation. A general expression for this force was derived valid for arbitrary curved trajectory. Unfortunately, these equations cannot be used for a beam passing through a single bending magnet because the scalar potential $\phi$ that enters the initial conditions for the equations of motion is not defined for a line-charge beam. We then derived the 3D expression for transverse force without renormalization, using the smallness of the beam size, $\sigma_{z}, \sigma_{\perp} \ll \kappa^{-1}$, and found an analytical expression for the steady-state case of a beam moving on a circular orbit. For particular examples of a spherically symmetric and a flat beams, our analytical expressions show an excellent agreement with direct calculation of the transverse force with the computer code PyCSR3D. We also calculated the transient effects at the entrance to and the exit from a bend, and observed that the transients deviate from the steady state profiles only on a relatively short distance, much shorter than the formation length of the CSR longitudinal wake field. This indicates that in practical applications, for short bunches, one can use simplified steady state expressions and ignore the transients.

\section{ACKNOWLEDGMENTS}

I am thankful to C. Mayes for helping with calculations of the transverse force with the computer code PyCSR3D. I would also like to thank Z. Huang, J. Tang, R. Li and E. Schneidmiller for stimulating discussions of various aspects of this work. This work was supported by the Department of Energy, Contract No. DE-AC03-76SF00515.

\section{APPENDIX A: HAMILTONIAN EQUATIONS}

In this Appendix we derive the Hamiltonian equations of particle motion in a given magnetic field that take into account the self-field of the beam. We will closely follow the derivation and the notation of the book [29]. The beam reference orbit (which is assumed to be planar) corresponding to the nominal momentum, $p_{0}=\sqrt{\left(\gamma_{0} m c\right)^{2}-m^{2} c^{2}}$, is given by a vectorial function $\boldsymbol{r}_{0}(s)$, where $s$ is the arc length measured along the trajectory. The tangential vector 
$\boldsymbol{\tau}=d \boldsymbol{r}_{0} / d s$ and the unit normal vector $\boldsymbol{n}$ are defined so that $d \boldsymbol{n} / d s=\kappa \boldsymbol{\tau}$ and $d \boldsymbol{\tau} / d s=-\kappa \boldsymbol{n}$, where $\kappa$ is the orbit curvature. The unit vectors $\hat{\boldsymbol{x}} \equiv \boldsymbol{n}, \hat{\boldsymbol{y}}$ and $\hat{\boldsymbol{s}} \equiv \boldsymbol{\tau}$ constitute a right-handed local coordinate system.
Using $s$ as an independent timelike variable, we start from the Hamiltonian (5.18) from [29] to which we add the transverse components of the vector potential, $A_{x}$ and $A_{y}$, and the electrostatic potential $\phi$,

$$
K=-(1+\kappa x)\left[\frac{1}{c^{2}}(H-e \phi)^{2}-m^{2} c^{2}-\left(\Pi_{x}-\frac{e A_{x}}{c}\right)^{2}-\left(\Pi_{y}-\frac{e A_{y}}{c}\right)^{2}\right]^{1 / 2}-\frac{e}{c} A_{s}(1+\kappa x) .
$$

The canonical coordinates here are: $t$ and $-H, x$ and $\Pi_{x}=p_{x}+e A_{x} / c, y$ and $\Pi_{y}=p_{y}+e A_{y} / c$, with $p_{x}$ and $p_{y}$ the components of the kinetic momentum, and $H=\gamma m c^{2}+e \phi$ the original Hamiltonian. The full kinetic momentum of the particle is $p=\sqrt{(H-e \phi)^{2} / c^{2}-m^{2} c^{2}}$, and its deviation from the nominal (constant) momentum $p_{0}$ is assumed small,

$$
\frac{p}{p_{0}}=1+\eta, \quad|\eta| \ll 1
$$

We now Taylor expand the square root in $K$ and replace $p^{2}$ by $p_{0}^{2}$ in the small terms that involve $\Pi_{x}$ and $\Pi_{y}$,

$$
K \approx-p_{0}(1+\kappa x)(1+\eta)\left[1-\frac{1}{2 p_{0}^{2}}\left(\Pi_{x}-\frac{e A_{x}}{c}\right)^{2}-\frac{1}{2 p_{0}^{2}}\left(\Pi_{y}-\frac{e A_{y}}{c}\right)^{2}\right]-\frac{e}{c} A_{s}(1+\kappa x) .
$$

It is convenient to introduce $\Delta H=H-\gamma_{0} m c^{2}$ and express $\eta$ through $\Delta H$,

$$
\eta=\frac{\sqrt{\left(\gamma_{0} m c^{2}+\Delta H-e \phi\right)^{2} / c^{2}-m^{2} c^{2}}}{p_{0}}-1 \approx \frac{\Delta H-e \phi}{c \beta_{0} p_{0}},
$$

with $\beta_{0}=p_{0} / \gamma m c$. The Hamiltonian (A3) then becomes

$$
K \approx-p_{0}(1+\kappa x)\left[1-\frac{1}{2 p_{0}^{2}}\left(\Pi_{x}-\frac{e A_{x}}{c}\right)^{2}-\frac{1}{2 p_{0}^{2}}\left(\Pi_{y}-\frac{e A_{y}}{c}\right)^{2}+\frac{\Delta H-e \phi}{c \beta_{0} p_{0}}\right]-\frac{e}{c} A_{s}(1+\kappa x) .
$$

Note that in this Hamiltonian, we assume the smallness of $(\Delta H-e \phi) / c \beta_{0} p_{0},\left(p_{x} / p_{0}\right)^{2}$ and $\left(p_{y} / p_{0}\right)^{2}$ (and we neglect higher powers and products of these quantities in what follows), but we do not simplify the factor $(1+\kappa x)$ to 1 even though the offset $x$ is supposed to be much smaller than the radius of curvature $|\kappa|^{-1}$.

We now split from the vector potential $A_{s}$ a part $A_{s}^{\text {ext }}$ that is responsible for the external magnetic field, $A_{s} \rightarrow A_{s}^{\text {ext }}+A_{s}$, where from now on $A_{s}$ denotes the contribution from the self field of the beam. The potential $A_{s}^{\text {ext }}$ is assumed to be independent of time and related to the external vertical magnetic field through the equation

$$
A_{s}^{\mathrm{ext}}(s, x)=-B_{y}^{\mathrm{ext}}(s) x\left(1-\frac{1}{2} \kappa x\right),
$$

with the orbit curvature

$$
\kappa(s)=\frac{e B_{y}^{\text {ext }}(s)}{p_{0} c}
$$

We then have

$$
\begin{aligned}
K= & -\frac{e}{c} A_{s}^{\mathrm{ext}}(1+\kappa x)-p_{0} \kappa x+\frac{e}{c \beta_{0}}(1+\kappa x)\left(\phi-\beta_{0} A_{s}\right) \\
& -(1+\kappa x) \frac{\Delta H}{c \beta_{0}}+\frac{1}{2 p_{0}}(1+\kappa x)\left(\Pi_{x}-\frac{e A_{x}}{c}\right)^{2} \\
& +\frac{1}{2 p_{0}}(1+\kappa x)\left(\Pi_{y}-\frac{e A_{y}}{c}\right)^{2},
\end{aligned}
$$

where we have dropped the constant $-p_{0}$ in the Hamiltonian.

We are now ready to formulate the equations of motion for this Hamiltonian. We start from the $t,-\Delta H$ variables,

$$
\frac{d t}{d s}=\frac{\partial K}{\partial(-\Delta H)}, \quad \frac{d(-\Delta H)}{d s}=-\frac{\partial K}{\partial t} .
$$

The first equation gives

$$
\frac{d t}{d s}=(1+\kappa x) \frac{1}{c \beta_{0}} \equiv v_{s}^{-1},
$$

with $v_{s}$ the rate of change of the coordinate $s$. Note that in this approximation we neglect the variation of the velocity 
due to the internal fields that changes the energy of the particle. This is a good approximation for relativistic particles. The second equation gives,

$$
\begin{aligned}
\frac{d \Delta H}{d s}= & \frac{e}{c \beta_{0}}(1+\kappa x)\left(\frac{\partial \phi}{\partial t}-\beta_{0} \frac{\partial A_{s}}{\partial t}\right) \\
& -(1+\kappa x)\left(\Pi_{x}-\frac{e A_{x}}{c}\right) \frac{e}{c p_{0}} \frac{\partial A_{x}}{\partial t} \\
& -(1+\kappa x)\left(\Pi_{y}-\frac{e A_{y}}{c}\right) \frac{e}{c p_{0}} \frac{\partial A_{y}}{\partial t} .
\end{aligned}
$$

To better understand the meaning of this equation we divide it by $d t / d s$ which gives the time derivative of $\Delta H$,

$$
\begin{aligned}
\frac{d \Delta H}{d t} & =e\left(\frac{\partial \phi}{\partial t}-\beta_{0} \frac{\partial A_{s}}{\partial t}\right)-p_{x} \frac{e \beta_{0}}{p_{0}} \frac{\partial A_{x}}{\partial t}-p_{y} \frac{e \beta_{0}}{p_{0}} \frac{\partial A_{y}}{\partial t} \\
& =e\left(\frac{\partial \phi}{\partial t}-\beta_{0} \frac{\partial A_{s}}{\partial t}\right)-e \beta_{x} \frac{\partial A_{x}}{\partial t}-e \beta_{y} \frac{\partial A_{y}}{\partial t} .
\end{aligned}
$$

This result is equivalent to the statement that the energy change per unit time is equal to $e \boldsymbol{v} \cdot \boldsymbol{E}$ with $\boldsymbol{E}$ given by Eq. (2). In the line-charge approximation where $\boldsymbol{\beta}=\boldsymbol{\tau}$ this equation reduces to Eq. (7).

We now consider the equations of motion in the horizontal plane. We have

$$
\frac{d x}{d s}=\frac{\partial K}{\partial \Pi_{x}}, \quad \frac{d \Pi_{x}}{d s}=-\frac{\partial K}{\partial x} .
$$

The first equation gives

$$
\frac{d x}{d s}=\frac{p_{x}}{p_{0}}
$$

and from the second equation one finds

$$
\begin{aligned}
\frac{d \Pi_{x}}{d s}= & \frac{e}{c}\left[\frac{\partial}{\partial x} A_{s}^{\mathrm{ext}}(1+\kappa x)\right]+\kappa p_{0} \\
& -\frac{e}{c \beta_{0}} \frac{\partial}{\partial x}[(1+\kappa x) \phi]+\frac{e}{c} \frac{\partial}{\partial x}\left[(1+\kappa x) A_{s}\right] \\
& +\kappa \frac{\Delta H}{c \beta_{0}}+\frac{e}{c p_{0}}(1+\kappa x)\left(\Pi_{x}-\frac{e A_{x}}{c}\right) \frac{\partial A_{x}}{\partial x} \\
& +\frac{e}{c p_{0}}(1+\kappa x)\left(\Pi_{y}-\frac{e A_{y}}{c}\right) \frac{\partial A_{y}}{\partial x},
\end{aligned}
$$

where we have neglected small terms of the order of $\kappa p_{x}^{2} / p_{0}$ and $\kappa p_{y}^{2} / p_{0}$. Expressing $\Pi_{x}$ through the momentum $p_{x}$ and the derivatives of the potentials through the components of the electromagnetic field according to the following equations,

$$
\begin{aligned}
B_{s} & =\frac{\partial A_{y}}{\partial x}-\frac{\partial A_{x}}{\partial y}, \quad E_{x}=-\frac{\partial \phi}{\partial x}-\frac{1}{c} \frac{\partial A_{x}}{\partial t}, \\
(1+\kappa x) B_{y} & =\frac{\partial A_{x}}{\partial s}-\frac{\partial A_{s}(1+\kappa x)}{\partial x},
\end{aligned}
$$

after some transformations this expression can be reduced to the following equation for the time derivative of $p_{x}$

$$
\begin{aligned}
\frac{d p_{x}}{d t}= & -c \beta_{0} p_{0} \frac{\kappa^{2} x}{1+\kappa x}+\frac{1}{\kappa^{-1}+x}(\Delta H-e \phi) \\
& +e E_{x}-e \beta_{0} B_{y}+\frac{e v_{y}}{c} B_{s} .
\end{aligned}
$$

We can now combine Eq. (A14) and (A17) to obtain a second order differential equation for $x$,

$$
\begin{aligned}
\frac{d^{2} x}{d s^{2}}= & \frac{d}{d s} \frac{p_{x}}{p_{0}}=\frac{1+\kappa x}{p_{0} c \beta_{0}} \frac{d p_{x}}{d t} \\
= & -\kappa^{2} x+\kappa \frac{\Delta H-e \phi}{c \beta_{0} p_{0}} \\
& +\frac{e}{c p_{0} \beta_{s}}\left(E_{x}-\beta_{0} B_{y}+\beta_{y} B_{s}\right),
\end{aligned}
$$

with $\beta_{s}=v_{s} / c$. Alternatively, one can also rewrite Eq. (A17) as

$\frac{d p_{x}}{d t}=-c \kappa \beta_{0} p_{0}+\frac{c \beta_{0} p_{0}}{\kappa^{-1}+x}+\frac{1}{\kappa^{-1}+x} \Delta H+\mathcal{F}$,

where $\mathcal{F}$ is the renormalized transverse force,

$$
\mathcal{F}=-\frac{1}{\kappa^{-1}+x} e \phi+e E_{x}-e \beta_{0} B_{y}+\frac{e v_{y}}{c} B_{s} .
$$

In the line-charge approximation, we replace $\kappa^{-1}+x \rightarrow \kappa^{-1}$, set $\beta_{0}=1$, and drop the last term that contains $v_{y}$. This leads to Eq. (9).

\section{APPENDIX B: TRANSVERE FORCE IN A LINE-CHARGE BEAM}

In this section we will show that the renormalized transverse force (10) is well defined for a line-charge beam on the beam orbit. In our calculations, we use the fact that locally at each point $s$, the beam orbit $\boldsymbol{r}_{0}(s)$ can be approximated by a circle of radius $\kappa^{-1}(s)$. This allows us to estimate the behavior of the integrands in various integrals in the limit $s^{\prime} \rightarrow s$ using the Taylor expansion of their numerator and the denominator.

We rewrite Eq. (10) in the following form,

$$
\begin{aligned}
\mathcal{F} & =-\kappa \phi+E_{x}-B_{y} \\
& =-\kappa\left(\phi-A_{s}\right)-\frac{\partial\left(\phi-A_{s}\right)}{\partial x}-\frac{1}{c} \frac{\partial A_{x}}{\partial t}-\frac{\partial A_{x}}{\partial s},
\end{aligned}
$$


where all the derivatives are evaluated on the reference orbit. We now split $\mathcal{F}$ into four terms,

$$
\frac{1}{e} \mathcal{F}=\mathcal{F}^{(1)}+\mathcal{F}^{(2)}+\mathcal{F}^{(3)}+\mathcal{F}^{(4)}
$$

with each $\mathcal{F}^{(i)}$ corresponding to one term on the right-hand side of Eq. (B1).

To simplify the notation, in what follows, we will omit the arguments in the distribution function, $\lambda\left(s^{\prime}-c t_{\text {ret }}\right) \rightarrow \lambda$, and replace $\boldsymbol{\tau}(s) \rightarrow \boldsymbol{\tau}, \boldsymbol{\tau}\left(s^{\prime}\right) \rightarrow \boldsymbol{\tau}^{\prime}, \boldsymbol{n}(s) \rightarrow \boldsymbol{n}, \boldsymbol{n}\left(s^{\prime}\right) \rightarrow \boldsymbol{n}^{\prime}$, $\boldsymbol{r}_{0}(s) \rightarrow \boldsymbol{r}_{0}, \boldsymbol{r}_{0}\left(s^{\prime}\right) \rightarrow \boldsymbol{r}_{0}^{\prime}$, and $\kappa(s) \rightarrow \kappa$. We will also denote by $\boldsymbol{R}$ the function $\boldsymbol{R}\left(s, s^{\prime}\right)=\boldsymbol{r}_{0}(s)-\boldsymbol{r}_{0}\left(s^{\prime}\right)$ and $R=\left|\boldsymbol{R}\left(s, s^{\prime}\right)\right|$. Then for the first component of the force we obtain,

$$
\frac{1}{e} \mathcal{F}^{(1)}=-\kappa\left(\phi-A_{s}\right)=-\kappa \int_{-\infty}^{\infty} \frac{d s^{\prime}}{R}\left(1-\boldsymbol{\tau}^{\prime} \cdot \boldsymbol{\tau}\right) \lambda .
$$

It contains the same integral as in Eq. (3) and as discussed in Sec. II does not have a singularity when $s^{\prime} \rightarrow s$. Indeed, for small $\left|s-s^{\prime}\right|$ we have $R \approx\left|s-s^{\prime}\right|$ and $1-\tau^{\prime} \cdot \tau \propto$ $\left|s-s^{\prime}\right|^{2}$, so in the limit $s^{\prime} \rightarrow s$ the integrand in Eq. (B3) tends to zero.

To evaluate $\mathcal{F}^{(2)}$ we need to calculate the $x$-derivative of the difference $\phi-A_{s}$ at $x=y=0$. Using the expression $t_{\text {ret }}\left(\boldsymbol{r}, \boldsymbol{r}^{\prime}, t\right)=t-\left|\boldsymbol{r}^{\prime}-\boldsymbol{r}\right| / c$ and the relations $\partial \boldsymbol{r} /\left.\partial x\right|_{x=y=0}=$ $\boldsymbol{n}$ and $\partial \boldsymbol{r} /\left.\partial s\right|_{x=y=0}=\boldsymbol{\tau}$ we find

$\left.\frac{\partial t_{\mathrm{ret}}}{\partial x}\right|_{x=y=0}=-\left.\frac{1}{c} \frac{\partial}{\partial x}\left|\boldsymbol{r}-\boldsymbol{r}_{0}^{\prime}\right|\right|_{x=y=0}=-\frac{1}{c} \frac{\boldsymbol{R} \cdot \boldsymbol{n}}{R}$,
$\left.\frac{\partial t_{\mathrm{ret}}}{\partial s}\right|_{x=y=0}=-\left.\frac{1}{c} \frac{\partial}{\partial s}\left|\boldsymbol{r}-\boldsymbol{r}_{0}^{\prime}\right|\right|_{x=y=0}=-\frac{1}{c} \frac{\boldsymbol{R} \cdot \boldsymbol{\tau}}{R}$.

This gives for the second term in the force,

$$
\begin{aligned}
\frac{1}{e} \mathcal{F}^{(2)}= & -\frac{\partial\left(\phi-A_{s}\right)}{\partial x} \\
= & \int_{-\infty}^{\infty} d s^{\prime} \frac{\boldsymbol{R} \cdot \boldsymbol{n}}{R^{3}}\left(1-\boldsymbol{\tau}^{\prime} \cdot \boldsymbol{\tau}\right) \lambda \\
& -\int_{-\infty}^{\infty} d s^{\prime} \frac{\boldsymbol{R} \cdot \boldsymbol{n}}{R^{2}}\left(1-\boldsymbol{\tau}^{\prime} \cdot \boldsymbol{\tau}\right) \lambda^{\prime} .
\end{aligned}
$$

In the last term of this equation we replaced $\partial_{t_{\text {ret }}} \lambda\left(s^{\prime}-c t_{\text {ret }}\right)=-c \lambda^{\prime}\left(s^{\prime}-c t_{\text {ret }}\right) \rightarrow-c \lambda^{\prime}$, where $\lambda^{\prime}$ is the derivative of the function $\lambda$ with respect to its argument. Both integrals on the right-hand side of this equation do not have singularities when $s^{\prime} \rightarrow s$ even though $R$ vanishes at $s^{\prime}=s$ - the zero of the denominators is compensated by the zero value of the numerators in the integrands. This is easily established using the estimates after Eq. (B3) together with $\boldsymbol{R} \cdot \boldsymbol{n} \propto\left|s-s^{\prime}\right|^{2}$ in the limit $s^{\prime} \rightarrow s$.
In the last two terms of Eq. (B1) we need the transverse component of the vector potential on the orbit. It is found from Eq. (1) by projecting it onto $\boldsymbol{n}$,

$$
A_{x}(s, t)=\int_{-\infty}^{\infty} d s^{\prime} \frac{\boldsymbol{\tau}^{\prime} \cdot \boldsymbol{n}}{R} \lambda .
$$

Calculating the time derivative of $A_{x}$ gives the third term of the force,

$$
\frac{1}{e} \mathcal{F}^{(3)}=\frac{\partial A_{x}}{\partial t}=-\frac{1}{c} \int_{-\infty}^{\infty} \frac{d s^{\prime}}{R} \boldsymbol{\tau}^{\prime} \cdot \boldsymbol{n} \lambda^{\prime} .
$$

Again, the vanishing $R$ in the integrand at $s^{\prime}=s$ is compensated by the term in the numerator $\boldsymbol{\tau}^{\prime} \cdot \boldsymbol{n} \propto$ $\left|s-s^{\prime}\right|$, making the integrand finite at $s^{\prime}=s$.

Finally, we need to calculate the $s$-derivative of $A_{x}$ in $\mathcal{F}^{(4)}$,

$$
\frac{\partial A_{x}}{\partial s}=\frac{\partial}{\partial s} \int_{-\infty}^{\infty} \frac{d s^{\prime}}{R} \boldsymbol{\tau}^{\prime} \cdot \boldsymbol{n} \lambda
$$

The combination $\boldsymbol{\tau}^{\prime} \cdot \boldsymbol{n} / R$ as a function of $s$ is discontinuous at $s=s^{\prime}$, where it jumps from the value equal to $\kappa$ a $s=s^{\prime}-0$ to $-\kappa$ at $s=s^{\prime}+0$. This discontinuity adds to the formal $s$-derivative of the integrand in (B8) a delta function, $-2 \kappa \delta\left(s-s^{\prime}\right)$, and gives the following result,

$$
\begin{aligned}
\frac{1}{e} \mathcal{F}^{(4)}= & -\frac{\partial A_{x}}{\partial s} \\
= & -2 \kappa \lambda(s-c t, t) \\
& +\int_{-\infty}^{\infty} \frac{d s^{\prime}}{R^{3}} \lambda\left[(\boldsymbol{R} \cdot \boldsymbol{\tau})\left(\boldsymbol{\tau}^{\prime} \cdot \boldsymbol{n}\right)-\kappa R^{2}\left(\boldsymbol{\tau}^{\prime} \cdot \boldsymbol{\tau}\right)\right] \\
& -\int_{-\infty}^{\infty} d s^{\prime} \frac{\left(\boldsymbol{\tau}^{\prime} \cdot \boldsymbol{n}\right)}{R^{2}}(\boldsymbol{R} \cdot \boldsymbol{\tau}) \lambda^{\prime},
\end{aligned}
$$

where we have used $\partial \boldsymbol{n} / \partial s=\kappa \boldsymbol{\tau}$. Analysis shows that both integrands in Eq. (B9) are finite when $s^{\prime} \rightarrow s$.

Combining Eqs. (B3), (B5), (B7), and (B9) and simplifying the resulting expression using the following relation,

$$
\begin{aligned}
& -(\boldsymbol{R} \cdot \boldsymbol{n})\left(\boldsymbol{\tau}^{\prime} \cdot \boldsymbol{\tau}\right)+(\boldsymbol{R} \cdot \boldsymbol{\tau})\left(\boldsymbol{\tau}^{\prime} \cdot \boldsymbol{n}\right) \\
& \quad=\boldsymbol{R} \cdot\left[-\boldsymbol{n}\left(\boldsymbol{\tau}^{\prime} \cdot \boldsymbol{\tau}\right)+\boldsymbol{\tau}\left(\boldsymbol{\tau}^{\prime} \cdot \boldsymbol{n}\right)\right]=-\boldsymbol{R} \cdot \boldsymbol{n}^{\prime},
\end{aligned}
$$

one arrives at the following final expression for the renormalized transverse force,

$$
\begin{aligned}
\frac{1}{e} \mathcal{F}= & -2 \kappa \lambda(s-c t)+\int \frac{d s^{\prime}}{R^{3}} \lambda\left[\boldsymbol{R} \cdot\left(\boldsymbol{n}-\boldsymbol{n}^{\prime}\right)-\kappa R^{2}\right] \\
& +\int \frac{d s^{\prime}}{R^{2}} \lambda^{\prime}\left[R \boldsymbol{\tau}^{\prime} \cdot \boldsymbol{n}-\boldsymbol{R} \cdot\left(\boldsymbol{n}-\boldsymbol{n}^{\prime}\right)\right] .
\end{aligned}
$$


Note that the curvature $\kappa$ in this equation is taken at the observation point, $\kappa=\kappa(s)$.

\section{APPENDIX C: COMPARISON WITH REF. [21]}

Equation (42) in Ref. [21] gives an expression for the transverse force in a beam with a uniform radial distribution. After correcting a typo in that equation (the function $\ln \xi$ in the integrand should be replaced by $\ln (\xi / R)$ ) and converting it to the notations of the current paper $(R=1 / \kappa)$, it takes the following form

$$
\begin{aligned}
\frac{1}{e} \bar{F}_{\perp}= & \lambda(u) \kappa\left[2 \ln \left(\frac{8}{\kappa a}\right)-3.91\right] \\
& +\kappa \int_{0}^{\infty} \ln (\zeta \kappa)\left[\frac{1}{3} \lambda^{\prime}(u-\zeta)-\lambda^{\prime}(u+\zeta)\right] d \zeta,
\end{aligned}
$$

where $a$ is the beam radius and the bar over $F_{\perp}$ denotes averaging over the transverse cross section of the beam. We now change the normalization of the argument of the logarithm function in the integrand from $\kappa^{-1}$ to $\sigma_{z}$,

$$
\begin{aligned}
\int_{0}^{\infty} & \ln (\zeta \kappa)\left[\frac{1}{3} \lambda^{\prime}(u-\zeta)-\lambda^{\prime}(u+\zeta)\right] d \zeta \\
= & \int_{0}^{\infty} \ln \left(\frac{\zeta}{\sigma_{z}}\right)\left[\frac{1}{3} \lambda^{\prime}(u-\zeta)-\lambda^{\prime}(u+\zeta)\right] d \zeta \\
& +\frac{4}{3} \lambda(u) \ln \left(\kappa \sigma_{z}\right),
\end{aligned}
$$

and write Eq. (C1) as

$$
\begin{aligned}
\frac{1}{e} \bar{F}_{\perp}= & \lambda(u) \kappa\left[2 \ln \left(\frac{\sigma_{z}^{2 / 3}}{\kappa^{1 / 3} a}\right)+0.25\right] \\
& +\kappa \int_{0}^{\infty} \ln \left(\frac{\zeta}{\sigma_{z}}\right)\left[\frac{1}{3} \lambda^{\prime}(u-\zeta)-\lambda^{\prime}(u+\zeta)\right] d \zeta .
\end{aligned}
$$

To establish connection of this equation with Eq. (42) we note that for a uniform radial distribution we can replace $\sigma_{\perp}$ in Eq. (41) by the beam radius $a$ and use $\mu(x, y)=1 /\left(\pi a^{2}\right)$ for $\sqrt{x^{2}+y^{2}} \leq a$, and $\mu=0$ otherwise. The averaging of the transverse force over the beam cross section means that $G(x, y)$ in Eq. (42) should be replaced by

$$
\begin{aligned}
\bar{G} & =\frac{1}{\pi a^{2}} \int d x d y G(x, y) \\
& =-\frac{2}{\pi a^{4}} \int_{0}^{a} r d r \int_{0}^{a} r^{\prime} d r^{\prime} \int_{0}^{2 \pi} d \theta \ln \left(\frac{r^{2}+r^{\prime 2}-2 r r^{\prime} \cos \theta}{a^{2}}\right) \\
& =0.5,
\end{aligned}
$$

where in the last step we used Eq. (41), converted the integral to the cylindrical coordinates and carried out the numerical integration. Adding this numerical value $\bar{G}=$ 0.5 to the remaining numerical factors in Eq. (42),
$2 \ln 2-2+\frac{1}{3} \ln 3=-0.25$, we see that Eq. (C3) [and hence $(\mathrm{C} 1)]$ indeed coincides with the averaged over the cross section force (42).

[1] A. W. Chao, Physics of Collective Beam Instabilities in High Energy Accelerators (Wiley, New York, 1993).

[2] F. Zimmermann and T. O. Raubenheimer, Longitudinal space charge in final-focus systems for linear colliders, Nucl. Instrum. Methods Phys. Res., Sect. A 390, 279 (1997).

[3] K. L. F. Bane and A. W. Chao, Longitudinal space charge effect in slowly converging or diverging relativistic beams, Phys. Rev. ST Accel. Beams 5, 104401 (2002).

[4] R. Talman, Novel Relativistic Effect Important in Accelerators, Phys. Rev. Lett. 56, 1429 (1986).

[5] E. P. Lee, Cancellation of the centrifugal space charge force, Part. Accel. 25, 241 (1990).

[6] Y. S. Derbenev and V.D. Shiltsev, Transverse Effects of Microbunch Radiative Interaction, Technical Report No. SLAC-PUB-7181, Stanford Linear Accelerator Center, Stanford, CA, USA, 1996.

[7] R. Li, Cancellation effects in CSR induced bunch transverse dynamics in bends, in Proceedings of EPAC 2002 (EPs-IGA, Geneva, 2002), p. 1365.

[8] J. B. Murphy, S. Krinsky, and R. L. Gluckstern, Longitudinal wakefield for synchrotron radiation, in Proc. IEEE Particle Accelerator Conference and International Conference on High-Energy Accelerators, Dallas, 1995 (IEEE, Piscataway, NJ, 1996), pp. 2980-2982, (IEEE Conference Record 95CH35843).

[9] Y. S. Derbenev, J. Rossbach, E. L. Saldin, and V.D. Shiltsev, Microbunch Radiative Tail-Head Interaction, DESY FEL Report No. TESLA-FEL 95-05 (Deutsches Elektronen-Synchrotron, Hamburg, Germany, 1995).

[10] J. B. Murphy, S. Krinsky, and R. L. Gluckstern, Longitudinal wakefield for an electron moving on a circular orbit, Part. Accel. 57, 9 (1997).

[11] E. L. Saldin, E. A. Schneidmiller, and M. V. Yurkov, On the coherent radiation of an electron bunch moving in an arc of a circle, Nucl. Instrum. Methods Phys. Res., Sect. A 398, 373 (1997).

[12] G. Stupakov and P. Emma, CSR wake for a short magnet in ultrarelativistic limit, in Proceedings of 8th European Particle Accelerator Conference (Paris, France, 2002), p. 1479 .

[13] C. Mayes and G. Hoffstaetter, Exact 1d model for coherent synchrotron radiation with shielding and bunch compression, Phys. Rev. ST Accel. Beams 12, 024401 (2009).

[14] D. Sagan, G. Hoffstaetter, C. Mayes, and U. Sae-Ueng, Extended one-dimensional method for coherent synchrotron radiation including shielding, Phys. Rev. ST Accel. Beams 12, 040703 (2009).

[15] W. Lou and G. H. Hoffstaetter, Coherent synchrotron radiation wake expressions with two bending magnets and simulation results for a multiturn energy-recovery linac, Phys. Rev. Accel. Beams 23, 054404 (2020).

[16] K. L. Bane, F.-J. Decker, Y. Ding, D. Dowell, P. Emma, J. Frisch, Z. Huang, R. Iverson, C. Limborg-Deprey, H. Loos, 
H.-D. Nuhn, D. Ratner, G. Stupakov, J. Turner, J. Welch, and $\mathrm{J}$. Wu, Measurements and modeling of coherent synchrotron radiation and its impact on the Linac Coherent Light Source electron beam, Phys. Rev. ST Accel. Beams 12, 030704 (2009).

[17] G. Geloni, J. Botman, O. Luiten, M. van der Wiel, M. Dohlus, E. Saldin, E. Schneidmiller, and M. Yurkov, Transverse self-fields within an electron bunch moving in an arc of a circle, Nucl. Instrum. Methods Phys. Res., Sect. A 522, 230 (2004).

[18] G. Geloni, E. Saldin, E. Schneidmiller, and M. Yurkov, Misconceptions regarding the cancellation of self-forces in the transverse equation of motion for an electron in a bunch, arXiv:physics/0310133.

[19] R. Li and Y. S. Derbenev, Discussions on the Cancellation Effect on a Circular Orbit, Particle accelerator. Proceedings, Conference, PAC'05, Knoxville, USA, May 16-20, 2005, Conf. Proc. C0505161, 1631 (2005).

[20] G. Stupakov and J. Tang, Calculation of the wake due to radiation and space charge forces in relativistic beams, Phys. Rev. Accel. Beams 24, 020701 (2021).

[21] G. Stupakov, Effect of centrifugal transverse wakefield for microbunch in bend, in Nonlinear and Collective Phenomena in Beam Physics 1998 Workshop, AIP Conference Proceedings No. 468, edited by S. Chattopadhyay,
M. Cornacchia, and C. Pelegrini (AIP, New York, 1999), pp. 334-47.

[22] Y. Cai and Y. Ding, Three-dimensional effects of coherent synchrotron radiation by electrons in a bunch compressor, Phys. Rev. Accel. Beams 23, 014402 (2020).

[23] R. Li and Y. Derbenev, Canonical formulations and cancellation effect in electrodynamics of relativistic beams on a curved trajectory, JLAB Technical Report No. JLABTN-02-054, 2002.

[24] Y. Cai, Coherent synchrotron radiation by electrons moving on circular orbits, Phys. Rev. Accel. Beams 20, 064402 (2017).

[25] G. Stupakov, Centripetal transverse wakefield in relativistic beam, arXiv:1901.10745.

[26] L. D. Landau and E. M. Lifshitz, The Classical Theory of Fields, 4th ed., Course of Theoretical Physics (Pergamon, London, 1979), Vol. 2 (Translated from Russian).

[27] C. Mayes, Computational approaches to coherent synchrotron radiation in two and three dimensions, J. Instrum. 16, P10010 (2021).

[28] C. Mayes, ChristopherMayes/pycsr3d: Pycsr3d version 0.2.0 (2021), 10.5281/zenodo.5496096.

[29] G. Stupakov and G. Penn, Classical Mechanics and Electromagnetism in Accelerator Physics, Graduate Texts in Physics (Springer International Publishing, New York, 2018). 